\title{
Authorship Attribution in Portuguese Using Character N-grams
}

\author{
Ilia Markov ${ }^{1}$, Jorge Baptista ${ }^{2}$, Obdulia Pichardo-Lagunas ${ }^{3}$ \\ ${ }^{1} \mathrm{CIC}$, Instituto Politécnico Nacional (IPN), Av. Juan de Dios Bátiz S/N, Del. Gus- \\ tavo A. Madero, 07738, Mexico City, Mexico \\ imarkov@nlp.cic.ipn.mx \\ ${ }^{2}$ Univ. Algarve/FCHS and INESC-ID Lisboa/L2F, Campus de Gambelas, P-8005- \\ 139, Faro, Portugal \\ jbaptis@ualg.pt \\ ${ }^{3}$ UPIITA, Instituto Politécnico Nacional (IPN), Av. Instituto Politécnico Nacional \\ 2580, Del. Gustavo A. Madero, 07340, Mexico City, Mexico \\ opichardola@ipn.mx
}

\begin{abstract}
For the Authorship Attribution (AA) task, character n-grams are considered among the best predictive features. In the English language, it has also been shown that some types of character n-grams perform better than others. This paper tackles the AA task in Portuguese by examining the performance of different types of character $n$-grams, and various combinations of them. The paper also experiments with different feature representations and machine-learning algorithms. Moreover, the paper demonstrates that the performance of the character n-gram approach can be improved by fine-tuning the feature set and by appropriately selecting the length and type of character $n$-grams. This relatively simple and language-independent approach to the AA task outperforms both a bag-of-words baseline and other approaches, using the same corpus.
\end{abstract}

Keywords: authorship attribution; character n-grams; Portuguese; stylometry; computational linguistics; machine learning

\section{Introduction}

The Authorship Attribution (AA) task aims at identifying the author of an anonymous target text given a predefined set of candidate authors and corresponding samples of their texts, deemed to be representative of their writing practices (style). In recent years, the AA task triggered an increasing interest due to its impact on marketing, security, and forensic linguistics, where it can help to limit the search space for the author of a text under investigation. From a machine-learning perspective, 
approaches to the AA task can be viewed as a multi-class, single-label classification problem, in which the set of class labels is known a priori. The challenge consists in modelling this classification task so that automatic methods can assign class labels (authors' names) to objects (text samples).

Character $n$-gram features have proved to be highly predictive not only for the AA task [13, 18, 35] but also for similar tasks, such as Author Profiling [20]. Character $n$-grams are language-independent features but they are able to capture lexical and syntactic information, as well as punctuation and capitalization information related with the authors' personal style [6,14]. Sapkota et al. [29] showed that, for the AA task in English, some categories of character $n$-grams perform better than others [29, p. 94]: "These categories are related to the three linguistic aspects hypothesized to be represented by character $n$-grams: morphosyntax (as represented by affix-like $n$-grams), thematic content (as represented by word-like $n$-grams) and style (as represented by punctuation-based $n$-grams)." Hence, the authors report that character $n$-grams that capture affixes and punctuation information, which can be related to morpho-syntactic and stylistic information, perform better than using all character $n$-grams.

This paper focuses on the AA task in the Portuguese language. Work on AA for the Portuguese language is still scarce [13,33,36,37], and several strategies have been put in practice with varying results. From the morphological point of view, Portuguese is a moderately rich language: besides a small set of productive prefixes and suffixes, most of these affixes can only be analysed by resourcing to the language's history; there is a relatively complex verbal inflection system, yielding around 70 inflected forms, several of them homographs; nominal and adjectival is limited to gender and number, within limited set of morphemes. Thus, the settings for the $n$-gram approach to the AA task as used for a language such as English is likely to yield different results in Portuguese. This makes it important to examine which types and/or combinations of character $n$-grams are the most predictive for the Portuguese language.

This paper shows that selecting optimal feature representation, a popular machinelearning algorithm, and combining different types of character $n$-grams, allows for improving previous results in AA task using the same Portuguese corpus. Moreover, appropriate tuning of the size of the feature data set can render significantly lighter the machine-learning processing with only slight variation of accuracy.

(i) Which types and length of character $n$-grams are optimal for the AA task in Portuguese?

(ii) Is it possible to enhance AA performance by selecting an appropriate parameters, that is, feature combinations, feature set size, etc. using only the training corpora?

(iii) Which feature representation and machine-learning algorithm provide the best results for this task?

(iv) Is the conclusion reported in [29], that the best performing model is based solely on affix and punctuation $n$-grams, valid for the Portuguese language? 


\section{Related Work}

Over the last decade, Authorship Attribution has become an important field of study in computational linguistics, among other factors because of its high stake applications in Social Media Forensics [4,5]. The PAN competition ${ }^{1}$ is a series of scientific events and shared tasks on digital text forensics, and it is one of the main fora regarding the AA and other related tasks. These tasks include Authorship Attribution proper, Authorship Verification (determine if two texts were written by the same author), Clustering (grouping documents by author) and Diarization (identify and group parts of a document written by the same author). Other tasks relate to Author Profiling (by gender, age or personality) and, more recently, Author Obfuscation.

A recent trend on AA and related tasks focused on cross-topic and cross-gender scenarios [35], which is a more realistic context to the development of practical applications of this task. In this section, a selection of the works on the Authorship Attribution task in both the English and Portuguese languages is briefly presented.

Many previous studies focused on finding stylometric features that represent the authors style $[18,26,30]$. [10] present an extensive list of the main features used in the AA task: word-based and punctuation-based features, either discarding or combining function words statistics, using stemming or lemmatization techniques, and term frequency-inverse document frequency $(t f-i d f)$. A popular approach involved using syntactic information extracted from texts: [37] based their approach on syntactic features such as subject, predicate, and accessories. In [10], Gómez-Adorno et al. showed that textual patterns obtained from shortest path walks over integrated syntactic graphs is a useful methodology for the AA task. Textual genre and length are key issues in AA. Most work on social media [2,3,33] have to tackle with the limited size of the texts.

Some linguistic-poor approaches are based on character $n$-grams. Many independent works have demonstrated that character $n$-grams are effective features for the AA task $[8,18,34]$. Character $n$-grams are predictive when used in isolation [8] or when combined with other stylometric features [25]. Several studies [22,35] investigated the impact of varying threshold values in single- and cross-topic AA conditions. The studies conclude that high threshold values are optimal for crosstopic AA. Finally, Sapkota et al. [29] introduced the notion, that this paper explores, that different types of $n$-grams may have differential predictive value for the AA task, showing that, for English, using affix+punctuation $n$-gram categories are more predictive than using all $n$-grams.

Different machine-learning techniques also perform in a varying way depending on a large number of factors [12]. Several works explore different machine-learning approaches to the AA task. Support Vector Machines (SVM) is a very popular machine-learning method in the field. [37] based their approach on syntactic features using various fusion methods with SVM. [28] showed that character-level convolutional neural networks outperform state-of-the-art approaches on four out of five examined datasets. Homem and Carvalho [13] explored fuzzy methods to

1 http://pan.webis.de 
determine authorship fingerprints in texts. Posadas-Durán et al. [24] showed that doc2vec-based feature representation outperforms the state-of-the-art approaches on the examined corpora.

Related work on the Portuguese Language is still scarce (see [1,19] for an overview). Pavelec et al. [23] used discourse connectors (mainly conjunctions) in Brazilian journalistic text as features to model the AA task. Sousa-Silva [32] experimented with different types of stylistic markers (POS-based, punctuation, word length, suffixes, pronouns, and conjunctions) drawing on a corpus of European Portuguese journalistic texts and using SVM, showing that simple quantitative data (word and sentence length, and punctuation), rather than more linguistic rich features, perform remarkably well. Work by Homem and Carvalho [13] also used character $n$-grams $(n=4)$ with a corpus of European Portuguese journalistic texts. Results bellow the threshold of $60 \%$ were reported. This corpus has been made available for this paper, so this is the most closely related data available for comparison. In one of the more recent work, [36] examined whether qualitative and quantitative analysis using SVM is an efficient approach to forensic cases of AA. [9] worked on gender classification (Author Profiling task) based on Twitter data in Portuguese. Silva et al. [33] focused on idiosyncratic usage on a corpus of social media data using SVM.

\section{Character N-gram Features}

In this work, the same, language-independent, character $n$-gram categories introduced by Sapkota et al. [29] are used. The original definitions for some of the categories are refined in order to make them more complete. This paper also experiments with character 4-grams, considering the usually larger word and affix length in Portuguese. The categories of character $n$-grams can be organized into three main super categories (affix-, word-, and punctuation-related $n$-grams). They are defined in Table 1. As an example, let us consider the following sample sentence (1):

(1) “Vejo-te na quarta-feira, está bem?”, respondeu o Pedro.

(“[I]'ll see you on Wednesday, is [that] okay?", replied Pedro.)

The character $n$-grams ( $n=3$ and 4 ) for the sample sentence (1) for each of the categories are shown in Tables 2 and 3, respectively. For clarity, spaces are represented by the underscore '.'.

Following the work by Sapkota et al. [29], three models of $n$-grams are examined:

1. All-untyped: when the categories of $n$-grams are ignored; any distinct $n$ gram is a different feature. This corresponds to the more common approach of extracting $n$-grams without classifying them into different categories.

2. All-typed: when $n$-grams of all available categories (affix+word+punct) are considered. Notice that instances of the same $n$-gram may refer to different features.

3. Affix+Punct: when the $n$-grams of the word category are excluded. 
Table 1

Categories of character $n$-grams introduced by Sapkota et al. [29].

\begin{tabular}{|c|c|}
\hline \multicolumn{2}{|c|}{ Affix character n-grams } \\
\hline prefix & $\begin{array}{l}\text { An } n \text {-gram that covers the first } n \text { characters of a word } \\
\text { that is at least } n+1 \text { characters long. }\end{array}$ \\
\hline suffix & $\begin{array}{l}\text { An } n \text {-gram that covers the last } n \text { characters of a word } \\
\text { that is at least } n+1 \text { characters long. }\end{array}$ \\
\hline space-prefix & $\begin{array}{l}\text { An } n \text {-gram that begins with a space and that does } \\
\text { not contain any punctuation mark. }\end{array}$ \\
\hline space-suffix & $\begin{array}{l}\text { An } n \text {-gram that ends with a space, that does not contain any } \\
\text { punctuation mark, and whose first character is not a space. }\end{array}$ \\
\hline \multicolumn{2}{|c|}{ Word character n-grams } \\
\hline whole-word & $\begin{array}{l}\text { An } n \text {-gram that encompasses all the characters of a word, } \\
\text { and that is exactly } n \text { characters long. }\end{array}$ \\
\hline mid-word & $\begin{array}{l}\text { An } n \text {-gram that contains } n \text { characters of a word that is at least } \\
n+2 \text { characters long, and that does not include neither the } \\
\text { first nor the last character of the word. }\end{array}$ \\
\hline multi-word & $\begin{array}{l}\text { An } n \text {-gram that spans multiple words, identified by the } \\
\text { presence of a space in the middle of the } n \text {-gram. }\end{array}$ \\
\hline \multicolumn{2}{|c|}{ Punctuation character n-grams (abbreviated as punct) } \\
\hline beg-punct & $\begin{array}{l}\text { An } n \text {-gram whose first character is a punctuation mark, } \\
\text { but the middle characters are not. }\end{array}$ \\
\hline mid-punct & $\begin{array}{l}\text { An } n \text {-gram whose middle character is a punctuation mark } \\
\text { (for } n=3 \text { ). }\end{array}$ \\
\hline end-punct & $\begin{array}{l}\text { An } n \text {-gram whose last character is punctuation mark, } \\
\text { but the first and the middle characters are not. }\end{array}$ \\
\hline
\end{tabular}

One of the main conclusions of Sapkota et al. [29] was that models based on affix+punct features were more efficient than models trained using all the features. In the current paper, these three models were applied in order to examine whether this conclusion is also valid for the Portuguese language.

Moreover, the performance of each category of character $n$-grams is examined separately, and the different models mentioned above are combined, aiming at identifying the most predictive stylometric feature combination for Portuguese.

\section{Experimental Settings}

In this section, the experimental settings are laid out. First the corpus here used is briefly described, in order to present the criteria adopted in building the two subsets used in the experiments, a balanced and an unbalanced subcorpus. Next, the method for defining a baseline is presented, using standard evaluation procedures and machine-learning algorithm (SVM), commonly used in this task. 
Table 2

Character $n$-grams $(n=3)$ per category for the sample sentence (1), where SC stands for Super Category.

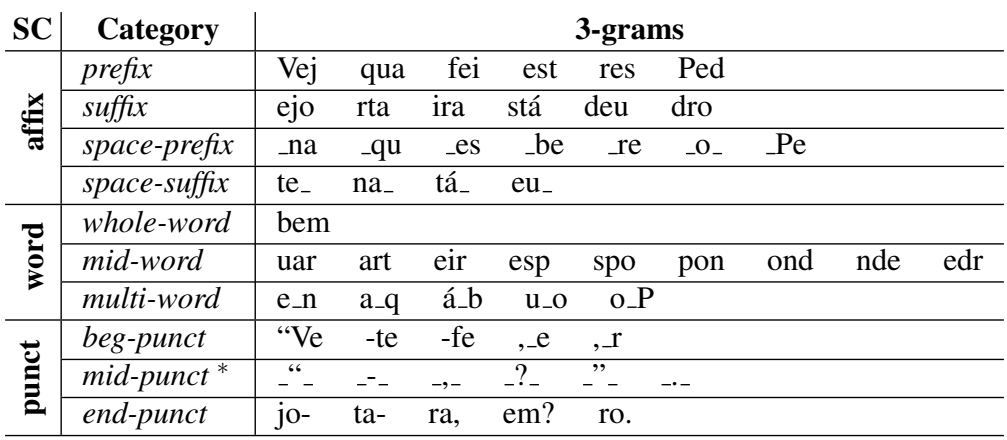

${ }^{*}$ In this work, punctuation marks are separated from adjacent words and from each other by space for this category. This enables to capture their frequency [22].

Table 3

Character $n$-grams $(n=4)$ per category for the sample sentence (1), where SC stands for Super Category.

\begin{tabular}{|c|c|c|c|c|c|c|c|}
\hline SC & Category & \multicolumn{6}{|c|}{ 4-grams } \\
\hline \multirow{4}{*}{ 気 } & prefix & quar & feir & resp & Pedr & & \\
\hline & suffix & arta & eira & ndeu & edro & & \\
\hline & space-prefix & _na & -qua & _est & _bem & _res & _Ped \\
\hline & space-suffix & stá_ & $\mathrm{deu}_{-}$ & & & & \\
\hline \multirow{3}{*}{ 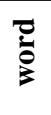 } & whole-word & Vejo & está & & & & \\
\hline & mid-word & uart & espo & spon & pond & onde & \\
\hline & multi-word $^{*}$ & te $\_n$ & na_q & tá_b & eu_o & $\mathrm{O} \_\mathrm{P}$ & \\
\hline \multirow{3}{*}{ 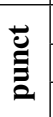 } & beg-punct & "Vej & - te & -fei & ,_es & _re & \\
\hline & mid-punct $^{* *}$ & $-"-$ & ${ }_{-}^{-}-$ & , $\quad$ ? & -"- & $+-\cdot$ & \\
\hline & end-punct & ejo- & rta- & ira, & pem? & dro. & \\
\hline
\end{tabular}

${ }^{*}$ In the case when the previous word is more than one character long, two characters are considered; otherwise, only one character is considered.

** This is the same as for character 3-grams (see Table 2).

\subsection{Corpus}

Experiments were conducted using a data set extracted from a large corpus composed of 5,167 newspaper articles (1,489,947 words) in Portuguese, which were retrieved from the on-line edition of the Público newspaper. This is the same set of texts that was used by Homem and Carvalho in their paper [13]. For this paper, though, the titles were included in the texts.

The texts in this corpus were written by 87 different authors on 16 distinct topics. The corpus here used is, therefore, a mixed-topic corpus. This corresponds to a realistic scenario, where the texts by candidate authors can be written either on the same topic or on different topics. The topics' classification is derived from the newspaper sections from hence they were retrieved. They include texts from national (26\%) to world news (16\%), including a local news section (8\%); topic-specific sections re- 
late to sport (20\%), economy (11\%) and culture (1\%); thematically mixed sections include: news highlights (6\%) and the last page (3\%) section, an opinion section, with texts from non-regular contributors' (3\%), as well as a chronicles section, with texts from regular contributors' (1\%); four of the 16 topics have less than 10 texts each. In spite of this topic distribution across the corpus, it should be noticed that, for the purpose of the AA task, some of the thematically mixed sections may also be considered of interest, as long as the number of texts per author is deem sufficient.

The corpus is highly unbalanced in terms of articles/author ratio, with an average of 59.5 texts per author, but only 34 authors having 60 or more texts, one author (chronicle) reaching 169 texts, while the least represented author has only 30 articles. The topic/author ratio is also quite unbalanced: for an average of 3.8 topics per author, only two authors address the maximum of 7 topics, while 9 only write about a single topic (and not always the same). Another issue is that there is a very short number of authors with a relevant number of texts in at least two different topics and, when this is the case, those topics do not always overlap. This situation raises considerable difficulties to cross-topic AA experiments with this corpus. For this paper, cross-topic AA is not addressed.

In the first phase of experiments, a balanced subset of the corpus was used, selecting only those authors who have at least 50 articles each. There were 50 authors with more than 50 articles per author. The corpus was then divided into two subsets and, in order to insure the reproducibility of the results, this splitting was based on the articles' ID, selecting the first 25 for training and the remaining 25 for evaluation. This corpus will be referred to as balanced.

In the second phase, the whole corpus was used. It was divided in a similar way as before, that is, the first half of articles per author were used for training and the second half for testing, using the texts' ID sequence. In case of an odd number of articles, the remaining text was added to the evaluation set. These settings were adopted in order to be able to compare this paper's results with those reported in [13], who proceed in the same way. This corpus will be referred to as unbalanced.

\subsection{Defining a Baseline}

The first set of experiments was carried out using the balance corpus. In order to better frame the results obtained from the different methods here applied to the AA task, the bag-of-words $(B o W)$ approach was defined as the baseline, which is a common procedure for this task, due to its language-independence and the fact that, in spite of its being a relatively simple and computationally inexpensive method, it already yields a strong (and challenging) baseline. In the BoW approach here used, features fed into the machine-learning algorithm are based on word frequency (punctuation marks are ignored).

In view of the size of the dataset, which comprises BoW 45,707 features, different frequency thresholds $(f r q)$ values were also experimented, in order to assess the impact of varying the size of the feature set in the performance of the machinelearning algorithms. In this way, frequency thresholds were tested using features 
with minimum $f r q \geqslant 25,50,75$, and 100 , that is, first using the whole set of features and then all the features with at least $25,50,75$, and 100 occurrences in the training corpus, thus progressively reducing the size of the feature set. According to [22] and [35], selecting an appropriate size of the feature set is important in cross-topic AA. Different threshold values were experimented in order to examine their impact under mixed-topic conditions in Portuguese.

Following Homem and Carvalho [13], who used the same corpus as this paper does, a 50\% training/testing corpus partition was adopted for the evaluation; only the training subcorpus is used in order to find the best parameters for the task at hand. This methodology also follows the practice of PAN international competitions on AA task, where the testing corpus is not made available to the competitors. Besides, experiments were also carried out using an adaptation of the 10 -fold cross evaluation method, where the training subcorpus is divided into 10 folds, only 9 are used to train the model and 1 is left out; the model is then evaluated on the testing subcorpus, and the process is repeated leaving another fold out; finally, the results of each training-testing stage are averaged.

Finally, two data representation methods have been compared, namely, the term frequency ( $t f$ ) described above; and a binary representation, which indicates just the presence or absence ( 1 or 0 ) of a feature in a given document. The tf-idf and normalized feature representation methods have also been experimented but they were dismissed as they did not show any positive effect on results. Table 4 shows the results from these experiments with a bag-of-word approach, varying the minimum frequency threshold, the feature representation, and the evaluation method.

Table 4

Baseline results in terms of accuracy (\%) using the bag-of-words (BoW) approach, with different frequency threshold values, different evaluation procedures, i.e. 10-fold cross-validation (10-fold) and 50\% training/testing evaluation (50\%-test), and different data representation methods, namely term frequency $(t f)$ vs. binary, using SVM algorithm. The top accuracy values in each experimental setting are shown in bold typeface.

\begin{tabular}{|c|c|c|c|c|c|}
\hline \multirow{2}{*}{$\begin{array}{l}\text { min. feature } \\
\text { frequency }\end{array}$} & \multicolumn{2}{|c|}{ tf } & \multicolumn{2}{|c|}{ binary } & \multirow{2}{*}{$\begin{array}{c}\text { N of } \\
\text { features }\end{array}$} \\
\hline & 10-fold & $50 \%$-test & 10-fold & $50 \%$-test & \\
\hline 0 (all features) & 59.52 & 51.36 & 70.40 & 61.84 & 45,707 \\
\hline 25 & 65.52 & 57.04 & 68.96 & 62.80 & 2,933 \\
\hline 50 & 64.08 & 55.84 & 67.36 & 60.16 & 1,554 \\
\hline 75 & 62.24 & 55.04 & 64.00 & 58.16 & 1,032 \\
\hline 100 & 59.04 & 50.80 & 60.24 & 52.48 & 749 \\
\hline
\end{tabular}

As one can see from Table 4, binary feature representation systematically outperforms term frequency ( $t f)$ data representation scheme, regardless of the examined threshold and of the evaluation procedures (10-fold cross-validation and 50\% training/test partition of the corpus). As expected, the 10-fold cross-validation setting also yields better results than the $50 \%$ training/test partition of the corpus.

Also as expected, the different frequency thresholds $(f r q)$ have a significant impact on the size of the feature set: for $f r q=25$, the 2,933 features corresponds to $6.42 \%$ of the entire data set; the number of features $(1,554)$ is almost halved for frq $=50$; 
the reduction in the feature data set $(1,032)$ is less pronounced $(66 \%)$ in the next threshold $(f r q=75)$; and even less important $(73 \%)$ for $f r q=100$.

Considering the size of the feature set for each frequency threshold, and comparing it with the accuracy obtained for each data representation method (tf/binary) and for the two evaluation scenarios (10-fold/50\%-test); it should be noticed that: (i) In the term frequency $(t f)$ settings, the best data size/accuracy combination is achieved with $f r q=25$; an important drop is observed when the entire data set is used instead $(6.00 \%$ and $5.68 \%$, in the 10 -fold and the 50\%-test scenarios, respectively); moving from $f r q=25$ to $f r q=50$ or from here to $f r q=75$ has only a minor effect in the performance of the classifiers, resulting in a reduction of accuracy slightly larger in the 10 -fold evaluation setting $(1.44 \%$ and $1.84 \%)$ than in the $50 \%$-test setting $(1.20 \%$ and $0.80 \%)$; another important drop (3.20\% and $4.24 \%$ ) occurs when selecting a $f r q=100$. This results can be interpreted in the sense that, when using the term frequency data representation method, low frequency words deteriorate the performance of the classifier, while important information is discarded if only highly frequent words are kept. (ii) In the binary feature representation setting, in 10-fold evaluation scenario the performance progressively decays (a drop of $1.44 \%$ from the best-performing all-features scenario to the $f r q=25$, and then, progressively, $1.60 \%$ to $f r q=50,3.36 \%$ to $f r q=75$ till $3.76 \%$ for $f r q=75$ ); on the other hand, in the $50 \%$-test evaluation setting, the all-features scenario actually yields slightly worst results than $f r q=25$; still, in both scenarios, the difference between the two best-performing thresholds is small $(1.44 \%$ in the 10 -fold setting, against $0.96 \%$ in the $50 \%$-test).

The best result (70.40\% accuracy) was achieved using binary feature representation in a 10 -fold cross-validation setting and taking all features into consideration. This could be interpreted as evidence that even low frequency words, usually associated with topic-specific information, provide useful information to the classifier. However, low frequency lexical features may be considered as too topic-specific, which may lead to unintended extraction of topic or domain information, instead of capturing the characteristics of the authors' style.

\section{Different Character N-gram Approaches}

Next, the $n$-gram methods were applied to the AA task using the same settings as described above, first in a 3-grams scenario and then in a 4-grams scenario. For each scenario, tree different models were built: (i) using only untyped $n$-grams; (ii) using only typed $n$-grams; and (iii) combining affix and punctuation $n$-grams, following the proposal of Sapkota et al. [29]. The 3-gram approach replicates previous experiments in the literature [20,22,29], while 4-grams were introduced to investigate wether it would be better suited for Portuguese, as it presents a moderately rich morphology. For each model, the minimum frequency threshold variation (from $\mathrm{frq}=0$ to 100 with step 25) was tested. 


\subsection{Character 3-gram and 4-gram Models}

Tables 5 and 6 present the results from the 3-gram and 4-gram scenarios, using the three different models presented above, and, for each model, the same frequency threshold values were tested. Again, the same feature representation and evaluation methods were examined. These tables also show, for each setting, the size of the feature set (number of features).

Table 5

Accuracy of character 3-gram models (untyped, typed, and affix+punctuation), across different frequency thresholds, using two feature representation methods and two evaluation procedures (this Table's headings are the same as those of Table 4). The size of the feature set in each specific setting is also provided. The best performing model for each type of feature set is highlighted in bold typeface. In case two models yield the same result, the one with the smaller number of features is selected.

\begin{tabular}{|c|c|c|c|c|c|c|}
\hline \multirow{2}{*}{ Model } & \multirow{2}{*}{$\begin{array}{l}\text { min. feature } \\
\text { frequency }\end{array}$} & \multicolumn{2}{|c|}{ tf } & \multicolumn{2}{|c|}{ binary } & \multirow{2}{*}{$\begin{array}{c}\mathrm{N} \text { of } \\
\text { features }\end{array}$} \\
\hline & & 10-fold & 50\%-test & 10-fold & 50\%-test & \\
\hline \multirow{5}{*}{ 总 } & 0 (all features) & 61.20 & 55.60 & 69.68 & 63.20 & 24,400 \\
\hline & 25 & 64.27 & 58.00 & 69.52 & 63.36 & 6,330 \\
\hline & 50 & 64.64 & 59.20 & 69.76 & 62.32 & 4,659 \\
\hline & 75 & 65.28 & 59.28 & 69.60 & 62.56 & 3,938 \\
\hline & 100 & 64.64 & 58.96 & 69.12 & 62.64 & 3,443 \\
\hline \multirow{5}{*}{ 芯 } & 0 & 61.92 & 55.44 & 70.64 & 64.16 & 27,686 \\
\hline & 25 & 64.80 & 59.28 & 70.32 & 64.32 & 7,283 \\
\hline & 50 & 64.88 & 59.60 & 70.72 & 63.36 & 5,413 \\
\hline & 75 & 65.36 & 60.00 & 70.32 & 63.60 & 4,559 \\
\hline & 100 & 65.36 & 59.84 & 69.92 & 63.20 & 3,965 \\
\hline \multirow{5}{*}{ 尝 } & 0 & 60.80 & 54.40 & 69.28 & 60.96 & 16,275 \\
\hline & 25 & 64.56 & 56.72 & 69.04 & 60.48 & 3,798 \\
\hline & 50 & 64.16 & 57.52 & 68.56 & 60.16 & 2,730 \\
\hline & 75 & 65.36 & 56.32 & 67.76 & 59.84 & 2,255 \\
\hline & 100 & 64.40 & 57.04 & 68.40 & 59.36 & 1,942 \\
\hline
\end{tabular}

As in the case of the BoW approach, binary feature representation always outperforms term frequency. The remaining of this paper will then focus on the results from binary feature representation, though the corresponding results for term frequency are also presented. Typed character 3-grams are slightly more predictive than either untyped or affix+punctuation, the later being showing the worst performance (though the difference is only marginal). The best 10 -fold cross-validation result was obtained with the threshold $f r q=50$. However, accuracy variation across thresholds in each model and even between different models is minimal.

Similarly to a 3-gram scenario, in a 4-gram scenario (Table 6) binary feature representation systematically provide higher results than term frequency. Also, the typed 4-gram model is slightly more predictive that both the untyped and the affix+punctation models. Again, the affix+punctation model is the worst performing.

The conclusion by Sapkota et al. [29] that using only affix+punctuation $n$-grams is more predictive than using all $n$-grams does not seem to be valid for the Portuguese language. This may indicate that word character $n$-gram category, which is 
Table 6

Accuracy of character 4-gram models (untyped, typed, and affix+punctuation), across different frequency thresholds, using two feature representation methods and two evaluation procedures (this Table's headings are the same as those of Table 4). The size of the feature set in each specific setting is also provided. The best performing model for each type of feature set is highlighted in bold typeface. In case two models yield the same result, the one with the smaller number of features is selected.

\begin{tabular}{|c|c|c|c|c|c|c|}
\hline \multirow{2}{*}{ Model } & \multirow{2}{*}{$\begin{array}{l}\text { min. feature } \\
\text { frequency }\end{array}$} & \multicolumn{2}{|c|}{ tf } & \multicolumn{2}{|c|}{ binary } & \multirow{2}{*}{$\begin{array}{c}\mathrm{N} \text { of } \\
\text { features }\end{array}$} \\
\hline & & 10-fold & 50\%-test & 10-fold & 50\%-test & \\
\hline \multirow{5}{*}{ 总 } & 0 (all features) & 60.96 & 54.64 & 69.28 & 66.16 & 92,646 \\
\hline & 25 & 67.04 & 60.88 & 70.48 & 65.92 & 15,547 \\
\hline & 50 & 67.12 & 61.76 & 70.24 & 65.60 & 10,535 \\
\hline & 75 & 66.96 & 61.44 & 70.48 & 65.44 & 8,125 \\
\hline & 100 & 66.88 & 60.56 & 70.48 & 64.72 & 6,717 \\
\hline \multirow{5}{*}{ 芯 } & 0 & 61.92 & 54.48 & 70.08 & 66.56 & 75,969 \\
\hline & 25 & 66.72 & 60.08 & 70.64 & 66.40 & 13,741 \\
\hline & 50 & 67.12 & 61.52 & 70.40 & 65.92 & 9,251 \\
\hline & 75 & 66.40 & 61.36 & 70.24 & 65.44 & 7,100 \\
\hline & 100 & 66.64 & 60.48 & 69.60 & 64.72 & 5,803 \\
\hline \multirow{5}{*}{ 总 } & 0 & 61.92 & 53.92 & 69.36 & 64.56 & 42,463 \\
\hline & 25 & 67.20 & 59.12 & 69.36 & 64.72 & 6,823 \\
\hline & 50 & 66.32 & 59.28 & 69.76 & 64.88 & 4,559 \\
\hline & 75 & 64.96 & 59.84 & 69.04 & 64.96 & 3,528 \\
\hline & 100 & 65.68 & 59.28 & 68.08 & 62.96 & 2,89 \\
\hline
\end{tabular}

considered to be more closely related to thematic content, should not be discarded when dealing with Portuguese. However, as the differences between models are only marginal, more experiments are required to verify this conclusion.

This is somehow strengthen by the fact that, in the BoW approach, low frequency words, which are related to topic-specific information, still contribute to AA accuracy in mixed-topic settings. However, as mentioned above, the results may be biased, since the approach may be capturing the topic information and not the style of the author. In the case of 4-grams, any threshold above 0 improves the results. According to [22,35], higher frequency threshold values provide better results in cross-topic AA. In the mixed-topic corpus used in this work, varying the frequency threshold does not seem to significantly improve the results; however, it allows for an important reduction of the size of the feature set without loss of accuracy.

Comparing the performance of 3- and 4-gram models, the difference is minimal using the 10-fold cross-validation evaluation method, while when using the 50\%test settings the results of the 4-gram models are approximately $3 \%-4 \%$ higher.

In view of these differences, the untyped 5-gram model was also examined in order to be able to establish the optimal length of character $n$-grams. Results are shown in Table 7. They are only slightly lower than those obtained when using 4-grams. In fact, the typed $n$-gram approach proposed by Sapkota et al. [29] is maximally efficient for $n$-gram models with the maximum length of 4 , since when using typed 5 -grams, many character $n$-grams are not captured by the proposed categories. Moving from a 4-gram to a 5-gram model does not seem to have much impact on the 
Table 7

Accuracy of untyped character 5-gram models, across different frequency thresholds, using two feature representation methods and two evaluation procedures (this Table's headings are the same as those of Table 4). The size of the feature set in each specific setting is also provided. The best performing model is highlighted in bold typeface. In case two models yield the same result, the one with the smaller number of features is selected.

\begin{tabular}{c|cc|cc|c}
\hline \multirow{2}{*}{$\begin{array}{c}\text { min. feature } \\
\text { frequency }\end{array}$} & \multicolumn{2}{|c|}{ tf } & \multicolumn{2}{c|}{ binary } & N of \\
\cline { 2 - 5 } & $\mathbf{1 0 - f o l d}$ & $\mathbf{5 0 \%}$-test & $\mathbf{1 0 - f o l d}$ & $\mathbf{5 0 \%}$-test & features \\
\hline 0 (all features) & 57.76 & 50.64 & 68.80 & 64.00 & 242,932 \\
25 & 67.12 & 58.16 & $\mathbf{7 0 . 1 6}$ & 64.40 & 24,553 \\
50 & $\mathbf{6 7 . 1 2}$ & $\mathbf{5 9 . 3 6}$ & 69.68 & $\mathbf{6 4 . 4 0}$ & 14,448 \\
75 & 65.76 & 58.24 & 69.04 & 64.16 & 10,173 \\
100 & 65.28 & 58.40 & 68.48 & 63.60 & 7,776 \\
\hline
\end{tabular}

Portuguese corpus, probably because average word length is larger than in English. Still, the size of the feature set significantly increases, which makes this a suboptimal approach. As the differences in the overall performance of 4- and 5-gram models are only marginal, more experiments may be necessary.

Comparing the results above (Tables 5-7) with the bag-of-words baseline approach (Table 4 ), one can see that most $n$-gram models outperform the BoW approach, even if the differences are small. Focusing only on the binary feature representation, the only cases when the best $n$-gram models were unable to yield better results than the best baseline models were: (i) the untyped 3-grams, 10-fold; (ii) the affix+punctuation 3-grams, 10-fold, 50\%-test; (iii) the affix+punctuation 4-grams, 10-fold; (iv) the untyped 5-grams, 10-fold. This confirms that the BoW approach is already quite a challenging benchmark for the AA task. In the next sections, different strategies will be put in place to improve the results reported so far.

\subsection{Exploring Typed and Untyped Character N-grams}

Based on the previous experiments, and focusing only on the binary feature representation and on the 10-fold cross-validation evaluation method, the threshold $f r q \geqslant 50$ was selected for the next experiments, since 3 out of the 6 best models were obtained using this threshold. By choosing this threshold an average reduction of $88 \%$ of the entire feature set is achieved.

Other experiments were carried out by cutting out the most frequently occurring words in the training corpus, namely by discarding the 50 most frequent words, and then by successively cutting $2 \%, 5 \%$, and $10 \%$ of the most frequent words. This strategy has proved to be helpful in related tasks, such as Author Profiling [20]. However, in the AA task, the most frequent words, which are stop-words for the most part, are considered of a great importance [14]. This conclusion is also valid for Portuguese, since discarding the most frequent $n$-gram features did not led to improvements in accuracy (for lack of space, results are not provided here).

Next, the contribution of each category of character $n$-grams is examined separately (Table 8 ). To do so, each category was discarded one by one and the performance 
of typed 3- and 4-grams was evaluated. If the result is improved (italics in Table 8), the examined category is not predictive; otherwise (bold typeface Table 8), it is a predictive category.

Table 8

Results in terms of accuracy (\%) per category using typed character 3- and 4-grams, threshold $\geqslant 50$, binary representation, and SVM algorithm. Three best predictive 3- and 4-gram categories are in bold typeface; three worst predictive 4-gram categories are in italics.

\begin{tabular}{|c|c|c|c|c|c|c|}
\hline \multirow{2}{*}{ Feature set } & \multicolumn{2}{|c|}{ 3-grams } & \multirow{2}{*}{$\begin{array}{c}\mathrm{N} \text { of } \\
\text { features }\end{array}$} & \multicolumn{2}{|c|}{ 4-grams } & \multirow{2}{*}{$\begin{array}{c}\mathrm{N} \text { of } \\
\text { features }\end{array}$} \\
\hline & 10-fold & $50 \%$-test & & 10-fold & 50\%-test & \\
\hline All categories & 70.72 & 63.36 & 5,413 & 70.40 & 65.92 & 9,251 \\
\hline All - prefix & 68.80 & 62.64 & 4,419 & 70.24 & 65.52 & 8,082 \\
\hline All - suffix & 69.76 & 63.52 & 4,768 & 70.80 & 66.24 & 8,205 \\
\hline All-space-prefix & 70.72 & 63.52 & 4,941 & 69.92 & 64.96 & 8,113 \\
\hline All - space-suffix & 70.08 & 63.52 & 5,186 & 70.32 & 66.32 & 8,629 \\
\hline All - whole-word & 70.24 & 63.28 & 5,303 & 70.48 & 66.00 & 9,088 \\
\hline All - mid-word & 69.76 & 62.80 & 3,447 & 70.40 & 65.04 & 6,265 \\
\hline All - multi-word & 70.48 & 63.12 & 4,806 & 70.80 & 65.92 & 7,708 \\
\hline All - beg-punct & 69.84 & 63.36 & 5,280 & 70.00 & 65.60 & 9,021 \\
\hline All - mid-punct & 70.32 & 63.12 & 5,400 & 70.32 & 65.84 & 9.238 \\
\hline All - end-punct & 70.56 & 63.76 & 5,167 & 70,96 & 65.76 & 8,910 \\
\hline
\end{tabular}

After establishing the best and worst performing 3- and 4-gram categories, a process of feature selection was undertaken. First, the most predictive 3-gram categories and their combinations were added to the model of All-typed character 4-grams. Results are presented in Table 9. The best model corresponds to combining All-typed character 4-grams with prefix 3-grams and middle-punctuation 3-grams categories. This best-performing model was then selected for the next step of feature selection. Next, the worst predictive 4-gram categories and their combinations were discarded from the best combination established in the previous experiment. The results are shown in Table 10. This strategy does not seem to improve the 10 -fold cross-validation accuracy, which indicates that, in these settings, all 4-gram categories contribute to the overall accuracy. However, there is a slight improvement in the 50\%-test settings when some 4-gram categories are discarded, namely and in decreasing order of accuracy: Best - multi-word (0.72\%), Best - end-punctuation (0.24\%), Best suffix-end-punctuation and Best - multi-word - end-punctuation (both $0.16 \%$ ). As results from this feature selection procedure did not improve accuracy, the strategy of combining different models was tested next.

Next, typed, untyped, 3- and 4-gram models were combined to find the most predictive stylometric feature combination. Typed and untyped $n$-grams are different features, since typed $n$-grams are tagged with the corresponding category. One of the reasons why the combination of typed and untyped $n$-grams can enhance the performance is that some typed $n$-grams, being divided into multiple categories, are discarded by the high threshold ( $f r q \geqslant 50)$, while untyped $n$-grams are still able to exceed this threshold; e.g., in the phrase "com compaixão" (with compassion) the untyped 3-gram com appears 2 times, but it corresponds to two distinct typed 3grams: one whole-word 3-gram and another prefix 3-gram. Moreover, punctuation 
Table 9

Results in terms of accuracy (\%) combining typed character 4-grams with the three best predictive 3gram categories and their combinations. Threshold $\geqslant 50$, binary representation, SVM algorithm.

\begin{tabular}{l|cc|c}
\hline \multicolumn{1}{c|}{ Feature set } & 10-fold & $\mathbf{5 0 \%}$-test & N of Features \\
\hline Best: All typed character 4-grams & 70.40 & $\mathbf{6 5 . 9 2}$ & 9,251 \\
\hline All + prefix 3-grams & 70.40 & 65.44 & 10,245 \\
All + suffix 3-grams & 70.56 & 65.44 & 9,896 \\
All + mid-word 3-grams & 70.56 & 65.28 & 11,217 \\
All + prefix + suffix 3-grams & 69.76 & 65.20 & 10,890 \\
All + prefix + mid-word 3-grams & $\mathbf{7 0 . 8 8}$ & 64.88 & 12,211 \\
All + suffix + mid-word 3-grams & 70.16 & 65.52 & 11,862 \\
All + prefix + suffix + mid-word 3-grams & 70.64 & 65.36 & 12,856 \\
\hline
\end{tabular}

Table 10

Results in terms of accuracy (\%) using the best feature combination from Table 9 (all + prefix + midword 3-grams) as baseline and discarding the worst predictive 4-gram categories (Table 8) and their combinations. Threshold $\geqslant 50$, binary representation, SVM algorithm.

\begin{tabular}{l|cc|c}
\hline \multicolumn{1}{c|}{ Feature set } & 10-fold & $\mathbf{5 0 \%}$-test & N of Features \\
\hline Best: All + prefix + mid-word 3-grams & 70.88 & 64.88 & 12,211 \\
\hline Best - suffix 4-grams & $\mathbf{7 0 . 8 8}$ & 64.56 & 11,165 \\
Best - multi-word 4-grams & 70.80 & $\mathbf{6 5 . 6 0}$ & 10,668 \\
Best - end-punct 4-grams & 70.72 & 65.12 & 11,870 \\
Best - suffix - multi-word 4-grams & 70.72 & 64.40 & 9,622 \\
Best - suffix - end-punct 4-grams & 70.72 & 65.04 & 10.824 \\
Best - multi-word - end-punct 4-grams & 70.48 & 65.04 & 10,327 \\
Best - suffix - multi-word - end-punct 4-grams & 70.64 & 64.08 & 9.281 \\
\hline
\end{tabular}

marks are separated from adjacent characters by space and included in the middlepunctuation category of typed $n$-grams, which produces different $n$-grams [22]; e.g., in the sample sentence (1), the instance em? constitutes just one untyped 3-gram, but it corresponds to two typed 3-grams: em? (end-punct) and _? (mid-punct). Results are shown in Table 11. Using untyped 3-and 4-grams in combination with typed 3 -grams yielded the best performance so far (72.16\%). Thus, this combination was selected for further experiments described in the next subsection.

\subsection{Further Experiments}

\subsubsection{Introducing Some Pre-Processing Steps}

Pre-processing has proved to be a useful strategy for AA and related tasks [11, $20,22,31]$. In this paper, two pre-processing steps were examined: (i) replacing digits and (ii) discarding text inside quotations, before extracting character $n$-gram features. The first method consists in replacing each digit by ' 0 ' (ex., 12,345 $\rightarrow$ 00,000 ) aiming at capturing the number format but not the actual number [22]; the second procedure aims at discarding information that does not reflect the author's writing style. Finally, the two proposed steps were combined. Results are shown in 
Table 11

Results in terms of accuracy (\%) combining typed and untyped $n$-grams with $n=3$ and 4 . Threshold $\geqslant 50$, binary representation, SVM algorithm.

\begin{tabular}{l|cc|c}
\hline \multicolumn{1}{c|}{ Model } & $\mathbf{1 0 - f o l d ~}$ & $\mathbf{5 0 \%}$-test & N of Features \\
\hline $\begin{array}{l}\text { Untyped 3-grams + untyped 4-grams } \\
\begin{array}{l}\text { Untyped 3-grams + untyped 4-grams + } \\
\text { typed 3-grams }\end{array}\end{array}$ & 71.68 & 66.00 & 15,194 \\
$\begin{array}{l}\text { Untyped 3-grams + untyped 4-grams + } \\
\text { typed 4-grams }\end{array}$ & 71.60 & 65.20 & 20,607 \\
\hline $\begin{array}{l}\text { Typed 3-grams + untyped 3-grams } \\
\text { Typed 3-grams + untyped 4-grams }\end{array}$ & 70.96 & 63.84 & 24,445 \\
Typed 3-grams + typed 4-grams & 71.52 & 65.36 & 10,072 \\
\hline $\begin{array}{l}\text { Typed 3-grams + typed 4-grams + } \\
\text { untyped 3-grams }\end{array}$ & 71.60 & 65.60 & 14,651 \\
$\begin{array}{l}\text { Typed 3-grams + typed 4-grams + } \\
\text { untyped 4-grams }\end{array}$ & 71.68 & $\mathbf{6 6 . 2 4}$ & 25,186 \\
\hline $\begin{array}{l}\text { Typed 4-grams + untyped 4-grams } \\
\text { Typed 4-grams + untyped 3-grams }\end{array}$ & 70.72 & 65.76 & 19,786 \\
$\begin{array}{l}\text { Typed 4-grams + typed 3-grams + } \\
\text { untyped 3-grams + untyped 4-grams }\end{array}$ & 71.68 & 66.00 & 13,910 \\
\hline
\end{tabular}

Table 12. The proposed pre-processing steps were unable to enhance the best 10fold cross-validation result achieved in the previous stage; however, they provided a slight improvement in the 50\%-test accuracy $(0.48 \%)$.

Table 12

Results in terms of accuracy (\%) after applying different pre-processing steps to the best feature combination from Table 11 . Threshold $\geqslant 50$, binary representation, SVM algorithm.

\begin{tabular}{l|cc|c}
\hline \multicolumn{1}{c|}{ Pre-processing } & 10-fold & $\mathbf{5 0 \%}$-test & N of Features \\
\hline Previous best: untyped 3-grams + & 72.16 & 65.20 & 20,607 \\
untyped 4-grams + typed 3-grams & & & \\
\hline Replacing digits & 71.76 & 65.68 & 20,299 \\
Discarding quotes & $\mathbf{7 2 . 1 6}$ & 65.28 & 20,566 \\
Replacing digits + discarding quotes & 71.68 & $\mathbf{6 5 . 6 8}$ & 20,260 \\
\hline
\end{tabular}

Other experiments were also carried out, namely, by converting all texts to lowercase and by replacing whole numbers by ' 0 ' (ex., 12,345 $\rightarrow 0$ ) and year mentions by 'YYYY' (ex., 2016 $\rightarrow$ YYYY). These experiments, however, did not lead to improvements in accuracy (for lack or space, the results are not provided here). In future work, the impact of other pre-processing steps will be investigated, such as discarding or anonymizing named entities [7].

\subsubsection{Using Other Machine-Learning Algorithms}

So far, WEKA's [12] implementation of Support Vector Machines (SVM) algorithm was used. This algorithm with default parameters is considered among the best for the AA task in both the English and the Portuguese languages [23, 33, 36, 37]. 
Multinomial Naive Bayes (NBM) classifier, which is known to provide high results for text classification tasks [15,22], was also examined. The J48 and Naive Bayes algorithms have also been examined but they were dismissed as they consistently showed lower results than the SVM algorithm.

Table 13 presents the results of comparing SVM with NBM performance trained on the best combination of $n$-gram categories (untyped 3-grams + untyped 4-grams + typed 3-grams) and without applying any pre-processing steps. In this case, NBM is $2.16 \%$ less accurate than SVM under 10 -fold cross-validation, but it slightly outperforms SVM in the 50\%-test setting. However, additional experiments using NBM classifier with various threshold values and models (untyped, typed, and affix+punctuation), as well as with different feature combinations, showed that the results obtained using NBM classifier are consistently lower than when using SVM (the results are not provided due to lack of space).

Table 13

Results in terms of accuracy (\%) using SVM and NBM machine-learning algorithms with the best combination of $n$-gram categories (untyped 3-grams + untyped 4-grams + typed 3-grams), a frequency threshold $f r q \geqslant 50$, with binary representation and without any pre-processing.

\begin{tabular}{l|cc|c}
\hline Machine-learning algorithm & 10-fold & $\mathbf{5 0 \%}$-test & N of Features \\
\hline SVM & $\mathbf{7 2 . 1 6}$ & 65.20 & 20,607 \\
NBM & 70.00 & $\mathbf{6 6 . 4 8}$ & 20,607 \\
\hline
\end{tabular}

\subsubsection{Using Unbalanced Corpus}

Finally, the best-performing model (untyped 3- and 4-grams + typed 3-grams, shown in Table 11) was applied to the unbalanced corpus (see Section 4.1, in fine) with the optimal parameters, selected from the best 10 -fold cross-validation results, in order to compare this approach with that of Homem and Carvalho [13]. The baseline experiment was also conducted using the optimal settings for the bag-of-words (BoW) approach: frequency threshold $f r q=0$ and binary feature representation (see Table 4). Table 14 shows the results obtained using SVM and NBM classifiers.

Table 14

Results in terms of accuracy (\%) when using the unbalanced corpus without any pre-processing: $\left(1^{s t}\right.$ row) the bag-of-words (BoW) baseline approach (frequency threshold $f r q \geqslant 0$, binary representation, and SVM algorithm); ( $2^{\text {nd }}$ and $3^{\text {rd }}$ rows) comparing SVM and NBM algorithms using the best feature combination from Table 11 with the frequency threshold $f r q \geqslant 50$ and binary representation.

\begin{tabular}{|c|c|c|c|}
\hline Unbalanced corpus & 10-fold & $50 \%$-test & $\mathbf{N}$ of Features \\
\hline Bag-of-words, SVM (baseline) & 62.53 & 57.27 & 68,429 \\
\hline $\begin{array}{l}\text { Untyped 3-grams + untyped 4-grams + } \\
\text { typed 3-grams (SVM) }\end{array}$ & 64.99 & 60.84 & 29,276 \\
\hline $\begin{array}{l}\text { Untyped 3-grams + untyped 4-grams + } \\
\text { typed 3-grams (NBM) }\end{array}$ & 59.98 & 57.16 & 29,276 \\
\hline
\end{tabular}

Using the best model with the unbalanced corpus yielded an accuracy of $64.99 \%$ under 10 -fold cross-validation and $60.84 \%$ in the $50 \%$-test setting. The SVM al- 
gorithm, when compared with the results obtained with the balanced corpus, shows a $7.17 \%$ and $4.36 \%$ drop in accuracy (under 10-fold cross-validation and in the $50 \%$-test setting, respectively), while the accuracy of the BoW baseline approach drops $7.87 \%$ and $4.57 \%$ (10-fold and 50\%-test, respectively). In spite of the sophisticated algorithm proposed by Homem and Carvalho [13], who also used the unbalanced corpus, their results were systematically below the $60 \%$ threshold. The BoW baseline approach with the optimal parameters selected in this paper showed results similar to those reported by these authors, while this paper approach based on (typed and untyped) character $n$-grams outperforms them.

\section{Conclusions}

The Authorship Attribution (AA) task aims at identifying the author of a text based on text samples from known authors. This paper demonstrated that character $n$ gram features are highly predictive for the AA task in Portuguese. It showed that the combination of character $n$-grams of different types and length, along with an appropriate selection of threshold values, feature representation, and machinelearning algorithm, allows one to achieve high performance in this task. The best result was achieved when training SVM classifier on the combination of untyped character 3-grams, untyped character 4-grams, and typed character 3-grams, using binary feature representation and considering only those features that occur at least 50 times in the training corpus. This language-independent approach, with a commonly used SVM algorithm, outperformed both the bag-of-words baseline and previous approaches using the same corpus.

Varying frequency threshold values did not lead to significant improvements on the results. However, it allowed an average reduction of $88 \%$ of the entire feature set without loss of accuracy. Moreover, the paper demonstrated that the parameters selected by 10 -fold cross-validation using only the training corpus provide nearoptimal results when used in the 50\%-test setting. The paper also showed that the conclusion of Sapkota et al. [29] that affix+punctuation character $n$-grams perform better than when using all $n$-grams is not valid for the Portuguese language. Still, more experiments using other corpora are required to verify this conclusion.

Finally, it was demonstrated that feature representation is an important aspect for the AA task in Portuguese. Binary feature representation, in all examined cases, provided higher results than term frequency (an average increase of $4.71 \%$ under 10 -fold cross-validation and of $5.44 \%$ in the $50 \%$-test setting using the balanced corpus). Therefore, in future work, alternative feature representation techniques will be tested, such as second order representation [17] or doc2vec-based feature representation [16]. The later has proved to provide good results for AA in English [24] and in related tasks [21]. The proposed approach will also be examined under cross-topic and cross-genre AA conditions.

\section{Acknowledgements}

This work was supported by the Mexican Government (Conacyt projects 240844 and 20161958, SIP-IPN 20171813, 20171344, and 20172008, SNI, COFAA-IPN) and by the Portuguese Government, through Fundação para a Ciência e a Tecnologia (FCT) with reference UID/CEC/50021/2013. The authors express their gratitude to 
J.P. Carvalho and his collaborators, who kindly made the corpus available for this paper.

\section{References}

[1] Almeida, D.: Atribuição de autoria com propósitos forenses. ReVEL Revista Virtual de Estudos de Linguagem, vol. 12, no. 23, 2014, pp. 148-186

[2] Barbon, S., Igawa, R., and Zarpelão, B.: Authorship verification applied to detection of compromised accounts on online social networks. Multimedia Tools and Applications, 2016, pp. 1-21

[3] Bhargava, M., Mehndiratta, P., and Asawa, K.: Stylometric analysis for authorship attribution on twitter. Proceedings of the $2^{\text {nd }}$ Intl. Conf. on Big Data Analytics, Springer, LNCS, vol. 8302, 2013, pp. 37-47

[4] Chaski, C.: Best Practices and Admissibility of Forensic Author Attribution. Journal of Lay and Policy, vol. 21, no. 2, 2013, pp. 1333-1376

[5] Coulthard, M. and Johnson, A. (Eds.): The Routledge Handbook of Forensic Linguistics, Routledge, 2010

[6] Daelemans, W.: Explanation in computational stylometry. Proceedings of the $14^{\text {th }}$ Intl. Conf. on Intelligent Text Processing and Computational Linguistics, CIC-Ling 2013, 2013, pp. 451-462

[7] Dias, F., Baptista, J., and Mamede, N.: Automated Anonymization of Text Documents. IEEE World Congress Computational Computational Inteligence/Intelligence Methods for NLP, 2016, pp.1287-1294

[8] Escalante, H., Solorio, T., and Montes-y-Gómez, M.: Local histograms of character n-grams for authorship attribution. Proceedings of ACL-HLT 2011, 2011, pp. 288-298

[9] Filho, J., Pasti, R., and de Castro, L.: Gender classification of twitter data based on textual meta-attributes extraction. New Advances in Information Systems and Technologies, Springer, 2016, vol. 444, pp. 1025-1034

[10] Gómez-Adorno, H., Sidorov, G., Pinto, D., Vilariño, D., and Gelbukh, A.: Automatic authorship detection using textual patterns extracted from integrated syntactic graphs. Sensors, vol. 16, no. 9, 2016

[11] Gómez-Adorno, H., Markov, I., Sidorov, G., Posadas-Durán, J.-P., SanchezPerez, M., and Chanona-Hernandez, L.: Improving feature representation based on a neural network for author profiling in social media texts. Computational Intelligence and Neuroscience, vol. 2016, 2016, 13 pages

[12] Hall, M., Frank, E., Holmes, G., Pfahringer, B., Reutemann, P., and Witten, I.: The WEKA data mining software: An update. SIGKDD Explorations, vol. 11 , no. 1,2009 , pp. 10-18 
[13] Homem, N. and Carvalho, J.P.: Web user identification with fuzzy fingerprints. Proceedings of the IEEE Intl. Conf. on Fuzzy Systems, IEEE Xplorer, 2011, pp. 2622-2629

[14] Kestemont, M.: Function words in authorship attribution. From black magic to theory? Proceedings of the $3^{\text {rd }}$ Workshop on Computational Linguistics for Literature, EACL 2014, 2014, pp. 59-66

[15] Kibriya, A., Frank, E., Pfahringer, B., and Holmes, G.: Multinomial naive Bayes for text categorization revisited. Proceedings of the $17^{\text {th }}$ Australian Joint Conf. on Advances in AI, AI 2004, 2005, pp. 488-499

[16] Le, Q. and Mikolov, T.: Distributed representations of sentences and documents. Proceedings of the $31^{\text {st }}$ Intl. Conf. on Machine Learning, ICML 2014, 2014, pp. 1188-1196

[17] López-Monroy, A., Montes-y-Gómez, M., Escalante, H., Villaseñor-Pineda, L., and Stamatatos, E.: Discriminative subprofile-specific representations for author profiling in social media. Knowledge-Based Systems, vol. 89, 2015, pp. 134-147

[18] Luyckx, K. and Daelemans, W.: Authorship attribution and verification with many authors and limited data. Proceedings of the $22^{\text {nd }}$ Intl. Conf. on Computational Linguistics, COLING 2008, 2008, pp. 513-520

[19] Marquilhas, R., and Cardoso, A.: O estilo do crime: A análise de texto em estilística forense. XXVII Encontro Nacional da Associação Portuguesa de Linguística - Textos selecionados, 2011, pp. 416-436

[20] Markov, I., Gómez-Adorno, H., and Sidorov, G.: Language- and subtaskdependent feature selection and classifier parameter tuning for author profiling. Working Notes Papers of the CLEF 2017 Evaluation Labs. CEUR Workshop Proceedings, vol. 1866, CLEF and CEUR-WS.org, 2017

[21] Markov, I., Gómez-Adorno, H., Posadas-Durán, J.-P., Sidorov, G., and Gelbukh, A.: Author profiling with doc2vec neural network-based document embeddings. Proceedings of the $15^{\text {th }}$ Mexican Intl. Conf. on Artificial Intelligence, MICAI 2016, vol. 10062, Part II, LNAI, Springer, 2017, pp. 117-131

[22] Markov, I., Stamatatos, E., and Sidorov, G.: Improving cross-topic authorship attribution: The role of pre-processing. Proceedings of the $18^{\text {th }}$ Intl. Conf. on Computational Linguistics and Intelligent Text Processing, CICLing 2017, LNCS, Springer, 2017, in press

[23] Pavelec, D., Justino, E., and Oliveira, L.: Author identification using stylometric features. Inteligencia Artificial, Revista Iberoamericana de Inteligencia Artificial, vol. 11, no. 36, 2007, pp. 59-66

[24] Posadas-Durán, J.-P., Gómez-Adorno, H., Sidorov, G., Batyrshin, I., Pinto, D., and Chanona-Hernández, L.: Application of the distributed document representation in the authorship attribution task for small corpora. Soft Computing, 2016, pp. 1-13 
[25] Qian, T., Liu, B., Chen, L., and Peng, Z.: Tri-training for authorship attribution with limited training data. Proceedings of ACL 2014, 2014, pp. 345-351

[26] Ramnial, H., Panchoo, S., and Pudaruth, S.: Authorship attribution using stylometry and machine learning techniques. Intelligent Systems Technologies and Applications, Springer, Advances in Intelligent Systems and Computing, vol. 384, 2016, pp. 113-125

[27] Rocha, A., Scheirer, W., Forstall, C., Cavalcante, T., Theophilo, A., Shen, B., Carvalho, A., and Stamatatos, E.: Authorship attribution for social media forensics. IEEE Transactions on Information Forensics and Security, 2016

[28] Ruder, S., Ghaffari, P., and Breslin, J.: Character-level and multi-channel convolutional neural networks for large-scale authorship attribution. arXiv preprint arXiv:1609.06686, 2016

[29] Sapkota, U., Bethard, S., Montes-y-Gómez, M., and Solorio, T.: Not all character n-grams are created equal: A study in authorship attribution. Proceedings of NAACL-HLT 2015, 2015, pp. 93-102

[30] Schwartz, R., Tsur, O., Rappoport, A., and Koppel, M.: Authorship attribution of micro-messages. Proceedings of EMNLP 2013, 2013, pp. 1880-1891

[31] Sidorov, G., Ibarra Romero, M., Markov, I., Guzman-Cabrera, R., ChanonaHernández, L., and Velásquez, F.: Detección automática de similitud entre programas del lenguaje de programación Karel basada en técnicas de procesamiento de lenguaje natural. Computación y Sistemas, vol. 20, no. 2, 2016, pp. 279-288

[32] Sousa-Silva, R., Sarmento, L., Grant, T., Oliveira, E., and Maia, B.: Comparing Sentence-Level Features for Authorship Analysis in Portuguese. Computational Processing of the Portuguese Language, Springer, LNAI vol. 6001, 2010, pp. 51-54

[33] Sousa-Silva, R., Laboreiro, G., Sarmento, L., Grant, T., Oliveira, E., and Maia, B.: 'twazn me!!! ;' Automatic authorship analysis of micro-blogging messages. Proceedings of the $16^{\text {th }}$ Intl. Conf. Application of Natural Language to Information Systems, Springer, vol. 6716, 2011, pp. 161-168

[34] Stamatatos, E.: Author identification using imbalanced and limited training texts. Proceedings of the $18^{\text {th }}$ Intl. Conf. on Database and Expert Systems Applications, DEXA 2007, 2007, pp. 237-241

[35] Stamatatos, E.: On the robustness of authorship attribution based on character n-gram features. Journal of Law \& Policy, vol. 21, no. 2, 2013, pp. 427-439

[36] Teles, L.: Atribuição de autoria em linguística forense: uma análise combinada para identificação de autor através do texto. Master thesis, Universidade de Lisboa, 2016

[37] Varela, P., Justino, E., Bortolozzi, F., and Oliveira, L.: A computational approach based on syntactic levels of language in authorship attribution. IEEE Latin America Transactions, vol. 14, no. 1, 2016, pp. 259-266 